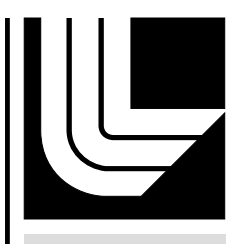

LA W REN CE LIVERMORE NATIONAL LABORATORY

A Mixed Formulation for the Brinkman Problem

P. S. Vassilevski, U. Villa

July 9,2012

Siam Journal on Numerical Analysis 
This document was prepared as an account of work sponsored by an agency of the United States government. Neither the United States government nor Lawrence Livermore National Security, LLC, nor any of their employees makes any warranty, expressed or implied, or assumes any legal liability or responsibility for the accuracy, completeness, or usefulness of any information, apparatus, product, or process disclosed, or represents that its use would not infringe privately owned rights. Reference herein to any specific commercial product, process, or service by trade name, trademark, manufacturer, or otherwise does not necessarily constitute or imply its endorsement, recommendation, or favoring by the United States government or Lawrence Livermore National Security, LLC. The views and opinions of authors expressed herein do not necessarily state or reflect those of the United States government or Lawrence Livermore National Security, LLC, and shall not be used for advertising or product endorsement purposes. 


\title{
A MIXED FORMULATION FOR THE BRINKMAN PROBLEM *
}

\author{
PANAYOT S. VASSILEVSKI ${ }^{\dagger}$ AND UMBERTO VILLA $\ddagger$
}

\begin{abstract}
The Brinkman model is a unified law governing the flow of a viscous fluid in cavity (Stokes equations) and in porous media (Darcy equations). In this work, we explore a novel mixed formulation of the Brinkman problem. Introducing the flow's vorticity as additional unknown, this formulation allows for a uniformly stable and conforming discretization by standard finite elements (Nédélec, Raviart-Thomas, piecewise discontinuous). The theoretical results are illustrated with numerical experiments. Based on our stability analysis of the problem in the $H(\operatorname{curl} ; \Omega)-H(\operatorname{div} ; \Omega)-$ $L^{2}(\Omega)$ norms, we derive, in a follow-up paper ([34]), a scalable block diagonal preconditioner which takes advantage of the auxiliary space AMG solvers for $H$ (curl) and $H$ (div) problems available in the preconditioning library hypre ([19]) developed at LLNL.
\end{abstract}

Key words. Brinkman problem; Stokes-Darcy coupling; saddle point problems; block preconditioners; algebraic multigrid.

Introduction. The Brinkman equations describe the flow of a viscous fluid in cavity and porous media. It was initially proposed in [1], [2] as a homogenization technique for the Navier-Stokes equations. Typical applications of this model are in underground water hydrology, petroleum industry, automotive industry, biomedical engineering, and heat pipes modeling.

Mathematically speaking the Brinkman model is a parameter-dependent combination of the Darcy and Stokes models. Since in real applications the number and the locations of the Stokes-Darcy interfaces might not be known a priori, the unified equations in the Brinkman model represent an advantage over the domain decomposition methods coupling the Darcy and the Stokes equations. However, the high variability in the PDE coefficients, that may take extremely large or small values, negatively affects the conditioning of the discrete problem which poses a substantial challenge for developing efficient preconditioners for this problem.

Another challenging aspect of the Brinkman model is the construction of a stable finite element discretization ([35]). In [24], it is proved that inf-sup compatible finite element for Stokes (Taylor-Hood, P2 - P0, Crouzeix-Raviart - P0, mini elements) will lead to non-convergent discretizations in the limit Darcy case (viscosity $\nu \rightarrow 0$ or inverse permeability $k \rightarrow \infty$ ) and that Raviart-Thomas elements for the discretization of the velocity field fail in the case $\nu \neq 0$, since they are not $H^{1}(\Omega)$ conforming. Numerous approaches have been proposed in the literature to address the numerical stability of various discretizations. Among those, in [12] and [13], the authors introduce jumps penalization on the normal component of the velocity field or on the pressure field to stabilize the Crouzeix-Raviart - P0 finite elements or P1 - P0 finite elements, respectively. In [15], an augmented Lagrangian approach and a least squares stabilization is explored in order to use inf-sup compatible Taylor-Hood elements also in the Darcy case, while in [24] and [18] high order non-conforming elements are investigated.

In the present paper, we analyze a mixed formulation of the Brinkman problem, in which we introduce the (scaled) flow vorticity as additional unknown. We prove

${ }^{*}$ This work was performed under the auspices of the U.S. Department of Energy by Lawrence Livermore National Laboratory under Contract DE-AC52-07NA27344.

† panayot@llnl.gov, Center for Applied Scientific Computing, Lawrence Livermore National Laboratory, P.O. Box 808, L-561, Livermore, CA 94551, U.S.A.

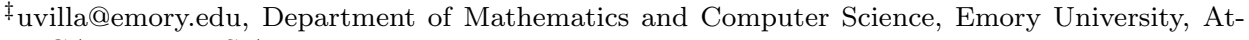
lanta, GA 30322, U.S.A. 
the well-posedness of the mixed formulation in the abstract framework of de Rham homology and Hilbert complexes, extending to the Brinkman problem the analysis of the mixed formulation of the Stokes problem in ([8], [4]). The numerical stability of the method is guaranteed by an appropriate choice of the finite elements spaces. The particular choice of Nédélec, Raviart-Thomas and piecewise discontinuous elements, in fact, reproduces the same embedding and mapping properties of the continuous spaces in the finite elements spaces. The linear system obtained after finite element discretization has a symmetric saddle point form. In contrast to the penalization methods for the Brinkman problem ([12], [13]), the proposed approach allows for a conforming discretization by standard finite elements. We also illustrate our analysis with numerical examples regarding the behavior of the discretization errors in the $H(\operatorname{curl} ; \Omega)$-norm of the vorticity, in the $H(\operatorname{div} ; \Omega)$-norm of the velocity and in the $L^{2}(\Omega)$-norm of the pressure. We numerically observe some suboptimal error behavior of the (scaled) vorticity in the Darcy limit, while velocity and pressure exhibit uniform error decay rates with respect to the inverse permeability coefficient $k(\mathbf{x})$.

We should point out on one possible disadvantage of the mixed formulation approach, namely that the Hodge decomposition holds only for particular sets of boundary conditions ([4]). On the other hand, the proven stability of our mixed discretization allows us to consider effective preconditioning techniques for the discrete saddle point problem. Following the approach in [25], we construct in [34] a block diagonal preconditioner with optimal convergence properties based on the stability analysis of the continuous problem. Such preconditioner has on its main diagonal the finite element matrices corresponding to the $H(\operatorname{curl} ; \Omega), H(\operatorname{div} ; \Omega)$, and $L^{2}(\Omega)$ norms involved in the stability estimates. To improve the efficiency of the preconditioner, we resort (in [34]) to the recently developed auxiliary space multigrid preconditioner for $H$ (div) and $H$ (curl) problems. The resulting (inexact) block-diagonal preconditioner appears to be robust with respect to constant and smoothly varying coefficient $k(\mathbf{x})$. The general case of fully robust (with respect to coefficient variation) preconditioning method is still an open problem. One possible approach is to develop appropriate adaptive (element-based) algebraic multigrid techniques that respect the entire de Rham complex on coarse levels. For some progress in that direction, we refer to [30], [23].

The remainder of the present paper is structured as follows. In Section 1, we briefly derive the mixed formulation of the Brinkman problem based on the Hodge Laplacian, and, in Section 2, we provide a stability estimate for the weak formulation. In Section 3, we address the numerical discretization of the mixed formulation with Nédélec, Raviart-Thomas and piecewise polynomial discontinuous finite element which leads to a large sparse saddle point linear system. In Section 4, we briefly outline our block-diagonal preconditioner. Finally, in Section 5 we present numerical results illustrating the error behavior of our discretization schemes for the case of constant coefficient, smoothly varying coefficient, and discontinuous coefficient.

1. Mixed formulation of the Brinkman Problem. We assume that $\Omega$ is a bounded simply connected domain in $\mathbb{R}^{d}$ with a regular (Lipschitz continuous) simply connected boundary $\partial \Omega$ that has well-defined (almost everywhere) unit outward normal vector $\mathbf{n} \in \mathbb{R}^{d}$. In what follows, sometimes we borrow some terminology from the finite element exterior calculus, e.g. from [8], without explicitly referring to this (or other) source. However, we keep this to a minimum to ensure that the presentation is self-contained. We also mostly consider the case $d=3$, as the analysis can be extended to the two-dimensional Brinkman problem with minimal variations. 
The generalized Brinkman problem reads

$$
\begin{cases}-\nu \Delta \mathbf{u}+k(\mathbf{x}) \mathbf{u}+\nabla p=\mathbf{f}(\mathbf{x}), & \forall \mathbf{x} \in \Omega \\ \operatorname{div} \mathbf{u}=g(\mathbf{x}), & \forall \mathbf{x} \in \Omega \\ \mathbf{u} \times \mathbf{n}=\mathbf{g}, & \text { on } \partial \Omega \\ -p+\nu \operatorname{div} \mathbf{u}=h, & \text { on } \partial \Omega,\end{cases}
$$

where $\nu \geq 0$ is the fluid viscosity and $k(\mathbf{x})$ is the inverse permeability of the medium. The challenge of this problems is when the coefficient $k=k(\mathbf{x})$ takes two extreme values $\mathcal{O}(1)$ and $\mathcal{O}(1 / \epsilon)$ in different parts of $\Omega$. In the part of the domain with $k=\mathcal{O}(1)$, the PDE behaves like a Stokes problem, whereas in the rest of the domain, it behaves like the Darcy equations.

In the present work, for simplicity, we assume natural boundary conditions on $\partial \Omega$. However, other set of boundary conditions, like the essential boundary conditions $\left(\mathbf{u} \cdot \mathbf{n}=u_{n}, \boldsymbol{\sigma} \times \mathbf{n}=\boldsymbol{\sigma}_{\tau}\right)$, can also be treated in a similar way. For the Hodge Laplacian, natural boundary conditions are also known in the literature as electric boundary conditions while the essential ones as magnetic boundary conditions due to the close relation with Maxwell's equations. In our work, we do not consider the case of full Dirichlet boundary condition, as the mixed formulation is harder to analyze; it leads to suboptimal discretization error behavior ([4]).

To obtain a mixed formulation of the Brinkman problem (1.1), we exploit the vector calculus identity

$$
\Delta \mathbf{u}=\nabla \operatorname{div} \mathbf{u}-\operatorname{curl} \operatorname{curl} \mathbf{u},
$$

and we define the (scaled) vorticity variable

$$
\boldsymbol{\sigma}=\varepsilon \operatorname{curl} \mathbf{u}, \varepsilon=\sqrt{\nu} .
$$

After some straightforward manipulations, the mixed formulation reads

$$
\begin{cases}\boldsymbol{\sigma}-\varepsilon \operatorname{curl} \mathbf{u}=0, & \forall \mathbf{x} \in \Omega \\ \varepsilon \operatorname{curl} \boldsymbol{\sigma}-\varepsilon^{2} \nabla \operatorname{div} \mathbf{u}+k(\mathbf{x}) \mathbf{u}+\nabla p=\mathbf{f}(\mathbf{x}), & \forall \mathbf{x} \in \Omega \\ \operatorname{div} \mathbf{u}=g(\mathbf{x}), & \forall \mathbf{x} \in \Omega \\ \mathbf{u} \times \mathbf{n}=\mathbf{g}, & \text { on } \partial \Omega \\ -p+\varepsilon^{2} \operatorname{div} \mathbf{u}=h, & \text { on } \partial \Omega .\end{cases}
$$

1.1. Functional spaces and orthogonal decompositions. We now introduce some notation used throughout the remainder of the paper. For vectorial functions $\mathbf{u}, \mathbf{v} \in \mathbf{L}^{2}(\Omega)=\left[L^{2}(\Omega)\right]^{d}$ and scalar functions $p, q \in L^{2}(\Omega)$, we define the inner products $(\mathbf{u}, \mathbf{v})=\int_{\Omega} \mathbf{u} \cdot \mathbf{v} d \Omega$ and $(p, q)=\int_{\Omega} p q d \Omega$. Similarly, we denote by $\|\mathbf{v}\|$ and $\|p\|$ the norms induced by the above inner products.

To come up with the weak formulation of the system (1.2), we introduce the functional spaces $\mathbf{Q}, \mathbf{R}$ and $W$, defined as

- $\mathbf{Q} \equiv H(\operatorname{curl} ; \Omega):=\left\{\boldsymbol{\sigma} \in L^{2}(\Omega) \mid \operatorname{curl} \boldsymbol{\sigma} \in \mathbf{L}^{2}(\Omega)\right\}$, equipped with the norm

$$
\|\boldsymbol{\tau}\|_{\mathbf{Q}}^{2}=\|\boldsymbol{\tau}\|^{2}+\|\operatorname{curl} \boldsymbol{\tau}\|^{2}
$$

- $\mathbf{R} \equiv H(\operatorname{div} ; \Omega):=\left\{\mathbf{u} \in L^{2}(\Omega) \mid \operatorname{div} \mathbf{u} \in L^{2}(\Omega)\right\}$, equipped with the norm

$$
\|\mathbf{v}\|_{\mathbf{R}}^{2}=\|\mathbf{v}\|^{2}+\|\operatorname{div} \mathbf{v}\|^{2} ;
$$


- $W \equiv L^{2}(\Omega)$, equipped with the norm

$$
\|q\|_{W}^{2}=\|q\|^{2} .
$$

We denote with $\mathbf{Q}^{*}, \mathbf{R}^{*}$, and $W^{*}$ the dual spaces of $\mathbf{Q}, \mathbf{R}$, and $W$, respectively. It is clear that in the case of essential (magnetic) boundary conditions, the respective spaces $\mathbf{Q}, \mathbf{R}$ are proper subsets of $H(\operatorname{curl} ; \Omega), H(\operatorname{div} ; \Omega) ; \mathbf{Q}, \mathbf{R}$ then consist of functions with vanishing tangential or normal boundary traces.

We need next the orthogonal decompositions of $H(\operatorname{curl} ; \Omega)$ and $H(\operatorname{div} ; \Omega)$ associated with the kernels of the respective differential operators. Such decompositions are of utmost importance in the stability analysis of the mixed problem, and for the derivation of the auxiliary space multigrid preconditioners for such spaces ([5], [6]).

The assumption that the domain $\Omega$ is simply connected with simply connected boundary, also called contractible (that is, any cycle in $\Omega$ is homologous to a point in $\Omega$ ), guarantees that de Rham sequence

$$
0 \rightarrow H^{1}(\Omega) \backslash \mathbb{R} \stackrel{\nabla}{\longrightarrow} H(\operatorname{curl} ; \Omega) \stackrel{\text { curl }}{\longrightarrow} H(\operatorname{div} ; \Omega) \stackrel{\operatorname{div}}{\longrightarrow} L^{2}(\Omega) \rightarrow 0
$$

is exact. In other words, the space of the k-harmonic forms has dimension 0 for all $k=$ $1,2,3$, or, equivalently, we have that range $(\nabla)^{\perp} \cap \operatorname{ker}(\operatorname{curl})=\{0\}$ and range(curl $)^{\perp} \cap$ $\operatorname{ker}(\operatorname{div})=\{0\}$. Therefore, we have that these nullspaces are defined as $\mathbf{X}=\{\boldsymbol{\tau} \in$ $\left.H(\operatorname{curl} ; \Omega) \mid \exists \psi \in H^{1}(\Omega): \boldsymbol{\tau}=\nabla \psi\right\}$ and $\mathbf{Y}=\{\mathbf{v} \in H(\operatorname{div} ; \Omega) \mid \exists \boldsymbol{\psi} \in H(\operatorname{curl} ; \Omega)$ : $\mathbf{v}=\operatorname{curl} \boldsymbol{\psi}$ \}. The original spaces admit the following orthogonal decompositions:

$$
H(\operatorname{curl} ; \Omega)=\mathbf{X} \oplus \mathbf{X}^{\perp}, \quad H(\operatorname{div} ; \Omega)=\mathbf{Y} \oplus \mathbf{Y}^{\perp},
$$

which imply the Poincaré inequalities

$$
\begin{aligned}
& \left\|\boldsymbol{\tau}^{\perp}\right\|_{H(\operatorname{curl} ; \Omega)} \leq \gamma\left\|\operatorname{curl} \boldsymbol{\tau}^{\perp}\right\|_{\mathbf{L}^{2}(\Omega)}, \quad \forall \boldsymbol{\tau}^{\perp} \in \mathbf{X}^{\perp}, \\
& \text { and }\left\|\mathbf{v}^{\perp}\right\|_{H(\operatorname{div} ; \Omega)} \leq \beta\left\|\operatorname{div} \mathbf{v}^{\perp}\right\|_{L^{2}(\Omega)}, \quad \forall \mathbf{v}^{\perp} \in \mathbf{Y}^{\perp},
\end{aligned}
$$

with constants $\gamma$ and $\beta$ which depend only on the domain $\Omega$. The orthogonal complement spaces are characterized as

$$
\begin{array}{ll}
\mathbf{X}^{\perp} & =\{\boldsymbol{\tau} \in H(\operatorname{curl} ; \Omega) \cap H(\operatorname{div} ; \Omega) \mid \operatorname{div} \boldsymbol{\tau}=0 \text { in } \Omega, \boldsymbol{\tau} \cdot \mathbf{n}=0 \text { on } \partial \Omega\}, \\
\text { and } & \mathbf{Y}^{\perp}=\left\{\mathbf{v} \in H(\operatorname{div} ; \Omega) \mid \exists \phi \in H_{0}^{1}(\Omega): \mathbf{v}=\nabla \phi\right\} .
\end{array}
$$

For more complete characterization of such spaces and for the orthogonal decompositions in the case of essential (magnetic) boundary conditions we refer, for example, to [33, Appendix A] or [26, 31].

1.2. Weak formulation. To obtain the weak formulation, we first multiply system (1.2) by $\boldsymbol{\tau} \in \mathbf{Q}, \mathbf{v} \in \mathbf{R}, q \in W$ and integrate over $\Omega$ :

$$
\left\{\begin{array}{l}
\int_{\Omega} \boldsymbol{\sigma} \cdot \boldsymbol{\tau} d \Omega-\int_{\Omega} \varepsilon(\operatorname{curl} \mathbf{u}) \cdot \boldsymbol{\tau} d \Omega=0, \quad \forall \boldsymbol{\tau} \in \mathbf{Q} \\
\int_{\Omega} \varepsilon(\operatorname{curl} \boldsymbol{\sigma}) \cdot \mathbf{v} d \Omega-\int_{\Omega} \varepsilon^{2}(\nabla \operatorname{div} \mathbf{u}) \cdot \mathbf{v} d \Omega+\int_{\Omega} k(\mathbf{x}) \mathbf{u} \cdot \mathbf{v} d \Omega+\int_{\Omega}(\nabla p) \cdot \mathbf{v} d \Omega= \\
=\int_{\Omega} \mathbf{f} \cdot \mathbf{v} d \Omega, \quad \forall \mathbf{v} \in \mathbf{R} \\
\int_{\Omega}(\operatorname{div} \mathbf{u}) q d \Omega=\int_{\Omega} g q d \Omega, \quad \forall q \in W .
\end{array}\right.
$$


By letting $\gamma_{T}(\boldsymbol{\tau})=\left(\mathbf{n} \times\left.\boldsymbol{\tau}\right|_{\partial \Omega}\right) \times \mathbf{n}, \gamma_{n}(\mathbf{v})=\left.\mathbf{n} \cdot \mathbf{v}\right|_{\partial \Omega}$ and exploiting some basic integration-by-parts identities ([27, Chapter 3$])$, the non-conforming terms become

$$
\begin{array}{r}
-\int_{\Omega} \varepsilon(\operatorname{curl} \mathbf{u}) \cdot \boldsymbol{\tau} d \Omega=-\int_{\Omega} \varepsilon \mathbf{u} \cdot(\operatorname{curl} \boldsymbol{\tau}) d \Omega+\int_{\partial \Omega} \varepsilon(\mathbf{u} \times \mathbf{n}) \cdot \gamma_{T}(\boldsymbol{\tau}) d S= \\
-\int_{\Omega} \varepsilon \mathbf{u} \cdot(\operatorname{curl} \boldsymbol{\tau}) d \Omega+\int_{\partial \Omega} \varepsilon \mathbf{g} \cdot \gamma_{T}(\boldsymbol{\tau}) d S,
\end{array}
$$

and

$$
\begin{aligned}
& -\int_{\Omega} \varepsilon^{2}(\nabla \operatorname{div} \mathbf{u}) \cdot \mathbf{v} d \Omega+\int_{\Omega}(\nabla p) \cdot \mathbf{v} d \Omega= \\
& =\int_{\Omega} \varepsilon^{2}(\operatorname{div} \mathbf{u})(\operatorname{div} \mathbf{v}) d \Omega-\int_{\Omega} p(\operatorname{div} \mathbf{v}) d \Omega+\int_{\partial \Omega}\left(p-\varepsilon^{2} \operatorname{div} \mathbf{u}\right) \gamma_{n}(\mathbf{v}) d S= \\
& =\int_{\Omega} \varepsilon^{2}(\operatorname{div} \mathbf{u})(\operatorname{div} \mathbf{v}) d \Omega-\int_{\Omega} p(\operatorname{div} \mathbf{v}) d \Omega-\int_{\partial \Omega} h \gamma_{n}(\mathbf{v}) d S .
\end{aligned}
$$

Therefore a weak solution $\boldsymbol{\sigma} \in \mathbf{Q}, \mathbf{u} \in \mathbf{R}$, and $p \in W$ of (1.2) satisfies the following variational problem

$$
\left\{\begin{array}{l}
\int_{\Omega} \boldsymbol{\sigma} \cdot \boldsymbol{\tau} d \Omega-\int_{\Omega} \varepsilon \mathbf{u} \cdot(\operatorname{curl} \boldsymbol{\tau}) d \Omega=-\int_{\partial \Omega} \varepsilon \mathbf{g} \cdot \gamma_{T}(\boldsymbol{\tau}) d S, \quad \forall \boldsymbol{\tau} \in \mathbf{Q} \\
\int_{\Omega} \varepsilon(\operatorname{curl} \boldsymbol{\sigma}) \cdot \mathbf{v} d \Omega+\int_{\Omega} \varepsilon^{2}(\operatorname{div} \mathbf{u})(\operatorname{div} \mathbf{v}) d \Omega+\int_{\Omega} k(\mathbf{x}) \mathbf{u} \cdot \mathbf{v} d \Omega-\int_{\Omega} p(\operatorname{div} \mathbf{v}) d \Omega= \\
=\int_{\Omega} \mathbf{f} \cdot \mathbf{v} d \Omega+\int_{\partial \Omega} h \gamma_{n}(\mathbf{v}) d S, \quad \forall \mathbf{v} \in \mathbf{R} \\
\int_{\Omega}(\operatorname{div} \mathbf{u}) q d \Omega=\int_{\Omega} g q d \Omega, \quad \forall q \in W .
\end{array}\right.
$$

The variational problem above can be written as an abstract saddle-point problem of the form

Problem 1.1. Find $(\boldsymbol{\sigma}, \mathbf{u}, p) \in \mathbf{Q} \times \mathbf{R} \times W$ such that

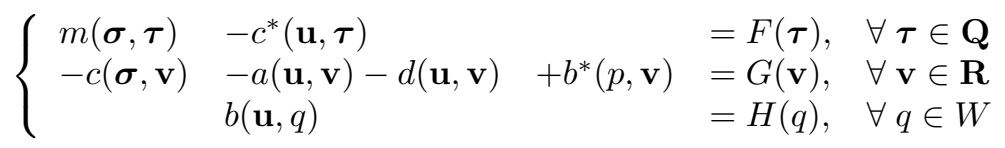

where

$$
\begin{array}{rlrl}
m(\boldsymbol{\sigma}, \boldsymbol{\tau}) & =(\boldsymbol{\sigma}, \boldsymbol{\tau}), & & \boldsymbol{\sigma}, \boldsymbol{\tau} \in \mathbf{Q} \\
c(\boldsymbol{\sigma}, \mathbf{v}) & =\varepsilon(\mathcal{C} \boldsymbol{\sigma}, \mathbf{v}), & \boldsymbol{\sigma} \in \mathbf{Q}, \mathbf{v} \in \mathbf{R}, \\
a(\mathbf{u}, \mathbf{v}) & =\varepsilon^{2}(\mathcal{B} \mathbf{u}, \mathcal{B} \mathbf{v}), & \mathbf{u}, \mathbf{v} \in \mathbf{R} \\
d(\mathbf{u}, \mathbf{v}) & =(k(\mathbf{x}) \mathbf{u}, \mathbf{v}), & \mathbf{u}, \mathbf{v} \in \mathbf{R} \\
b(\mathbf{u}, q) & =(\mathcal{B} \mathbf{u}, q), & & \mathbf{u} \in \mathbf{R}, q \in W
\end{array}
$$

Here $\mathcal{C}, \mathcal{B}$ denote some differentiation operators (curl and div), and $F \in \mathbf{Q}^{*}, G \in \mathbf{R}^{*}$, $H \in W^{*}$ are bounded functionals that take into account volume forces and boundary conditions. 
2. Well-posedness of the mixed variational formulation. Before studying the well-posedness of the mixed formulation of the Brinkman problem (1.1), we recall the following classical result due to Babuška ([9]).

TheOREM 2.1. Let $\mathfrak{B}: X \times X \rightarrow \mathbb{R}$ be a symmetric bounded bilinear form on a Hilbert space $X$ which satisfies the inf-sup condition

$$
\alpha:=\inf _{0 \neq y \in X} \sup _{0 \neq x \in X} \frac{\mathfrak{B}(x, y)}{\|x\|_{X}\|y\|_{X}}>0 .
$$

Then the problem of finding $\mathfrak{B}(x, y)=\mathfrak{F}(y)$ for all $y \in X$ is well-posed: it has a unique solution $x$ for each $\mathfrak{F} \in X^{*}$, and the following a priori estimate holds

$$
\|x\|_{X} \leq \frac{1}{\alpha}\|\mathfrak{F}\|_{X^{*}}
$$

In what follows, we assume that the inverse permeability coefficient $k(\mathbf{x})$ belongs to $L^{\infty}(\Omega) \cap L^{2}(\Omega)$, and we let $k_{\max }<+\infty$ be a constant such that

$$
0 \leq k(\mathbf{x}) \leq k_{\max } \text { almost everywhere in } \Omega .
$$

Since (by assumption) the inverse permeability $k(\mathbf{x})$ and the viscosity $\varepsilon^{2}$ can not both vanish at the same time, there exist constants $\kappa_{\min }>0$ and $\kappa_{\max }<+\infty$, such that

$$
0<\kappa_{\min }=k_{\min }+\varepsilon^{2} \leq k(\mathbf{x})+\varepsilon^{2} \leq k_{\max }+\varepsilon^{2}=\kappa_{\max } .
$$

For the stability analysis of Problem 1.1, we use the following weighted norms

$$
\begin{gathered}
\|\boldsymbol{\tau}\|_{\mathbf{Q}_{w}}^{2}=\|\boldsymbol{\tau}\|^{2}+\frac{\varepsilon^{2}}{\kappa_{\max }}\|\mathcal{C} \boldsymbol{\tau}\|^{2} \\
\|\mathbf{v}\|_{\mathbf{R}_{w}}^{2}=\kappa_{\min }\|\mathbf{v}\|_{\mathbf{R}}^{2}=\kappa_{\min }\|\mathbf{v}\|^{2}+\kappa_{\min }\|\mathcal{B} \mathbf{v}\|^{2} \\
\|q\|_{W_{w}}^{2}=\frac{1}{\kappa_{\max }}\|q\|^{2} .
\end{gathered}
$$

REMARK 2.1. The weighted space $\mathbf{Q}_{w}$ can be interpreted as intersection of Hilbert spaces, that is $\mathbf{Q}_{w}=\mathbf{L}^{2}(\Omega) \bigcap \frac{\varepsilon^{2}}{\kappa_{\max }} H(\operatorname{curl} ; \Omega)$. This space is equal to $H(\operatorname{curl} ; \Omega)$ as a set, but the corresponding norm approaches the $\mathbf{L}^{2}(\Omega)$ norm as $\frac{\varepsilon^{2}}{\kappa_{\max }}$ tends to zero. In other words, the smaller this ratio, the less control we have on the $\mathbf{L}^{2}(\Omega)$ norm of the curl of functions in $\mathbf{Q}_{w}$. For a detailed characterization of the intersection of Hilbert spaces and of their dual spaces we refer, for example, to [11, Chapter 2] and [25].

The stability of the mixed formulation of the Brinkman problem, which is our central result, is stated below.

Theorem 2.2. If $\varepsilon \geq 0$ and $k(\mathbf{x}) \in L^{\infty}(\Omega) \cap L^{2}(\Omega), 0<\kappa_{\min } \leq k(\mathbf{x})+\varepsilon^{2} \leq$ $\kappa_{\max }$ almost everywhere in $\Omega$, then for given continuous linear functionals $F \in \mathbf{Q}_{w}^{*}$, $G \in \mathbf{R}_{w}^{*}, H \in W_{w}^{*}$ the generalized Brinkman problem (1.1) admits a unique solution and the following a priori estimate holds:

$$
\|\boldsymbol{\sigma}\|_{\mathbf{Q}_{w}}^{2}+\|\mathbf{u}\|_{\mathbf{R}_{w}}^{2}+\|p\|_{W_{w}}^{2} \leq C(\Omega)\left(\|F\|_{\mathbf{Q}_{w}^{*}}^{2}+\|G\|_{\mathbf{R}_{w}^{*}}^{2}+\|H\|_{W_{w}^{*}}^{2}\right),
$$


where $C(\Omega)$ is a constant depending only on the domain.

Proof. The variational mixed formulation of the Brinkman problem (1.1) can be written in the form of Theorem 2.1, where $X=\mathbf{Q}_{w} \times \mathbf{R}_{w} \times W_{w}$ and $\mathfrak{B}$ denotes the symmetric bilinear form

$$
\begin{aligned}
\mathfrak{B}(\boldsymbol{\sigma}, \mathbf{u}, p ; \boldsymbol{\tau}, \mathbf{v}, q) & =(\boldsymbol{\sigma}, \boldsymbol{\tau})-\varepsilon(\mathbf{u}, \mathcal{C} \boldsymbol{\tau}) \\
& -\varepsilon(\mathcal{C} \boldsymbol{\sigma}, \mathbf{v})-(k(\mathbf{x}) \mathbf{u}, \mathbf{v})-\varepsilon^{2}(\mathcal{B} \mathbf{u}, \mathcal{B} \mathbf{v})+(p, \mathcal{B} \mathbf{v})+(\mathcal{B} \mathbf{u}, q),
\end{aligned}
$$

and $\mathfrak{F}(\boldsymbol{\tau}, \mathbf{v}, q)=F(\boldsymbol{\tau})+G(\mathbf{v})+H(q)$.

Then, Lemmas 2.3 and 2.4 imply Theorem 2.2, by establishing respectively the boundedness of $\mathfrak{B}$ and the inf-sup condition (2.1) required by Babuška's theorem (Theorem 2.1).

Lemma 2.3. Under the hypothesis of Theorem 2.2, there exists a constant $M$ such that for any $(\boldsymbol{\sigma}, \mathbf{u}, p),(\boldsymbol{\tau}, \mathbf{v}, q) \in \mathbf{Q}_{w} \times \mathbf{R}_{w} \times W_{w}$

$|\mathfrak{B}(\boldsymbol{\sigma}, \mathbf{u}, p ; \boldsymbol{\tau}, \mathbf{v}, q)| \leq$

$$
M \frac{\kappa_{\max }}{\kappa_{\min }}\left(\|\boldsymbol{\sigma}\|_{\mathbf{Q}_{w}}^{2}+\|\mathbf{u}\|_{\mathbf{R}_{w}}^{2}+\|p\|_{W_{w}}^{2}\right)^{\frac{1}{2}}\left(\|\boldsymbol{\tau}\|_{\mathbf{Q}_{w}}^{2}+\|\mathbf{v}\|_{\mathbf{R}_{w}}^{2}+\|q\|_{W_{w}}^{2}\right)^{\frac{1}{2}} .
$$

Proof. Simple applications of Cauchy-Schwarz inequalities show that the desired boundedness holds with $M=2$. Indeed, we have

$$
\begin{gathered}
|\mathfrak{B}(\boldsymbol{\sigma}, \mathbf{u}, p ; \boldsymbol{\tau}, \mathbf{v}, q)| \leq\|\boldsymbol{\sigma}\|\|\boldsymbol{\tau}\|+\varepsilon\|\mathbf{u}\|\|\mathcal{C} \boldsymbol{\tau}\|+\varepsilon\|\mathcal{C} \boldsymbol{\sigma}\|\|\mathbf{v}\|+ \\
\quad+k_{\max }\|\mathbf{u}\|\|\mathbf{v}\|+\varepsilon^{2}\|\mathcal{B} \mathbf{u}\|\|\mathcal{B} \mathbf{v}\|+\|p\|\|\mathcal{B} \mathbf{v}\|+\|\mathcal{B} \mathbf{u}\|\|q\| \leq \\
\leq\left(\|\boldsymbol{\sigma}\|^{2}+\frac{\varepsilon^{2}}{\kappa_{\max }}\|\mathcal{C} \boldsymbol{\sigma}\|^{2}+\left(\kappa_{\max }+k_{\max }\right)\|\mathbf{u}\|^{2}+\left(\kappa_{\max }+\varepsilon^{2}\right)\|\mathcal{B} \mathbf{u}\|^{2}+\frac{1}{\kappa_{\max }}\|p\|^{2}\right)^{\frac{1}{2}} \\
\left(\|\boldsymbol{\tau}\|^{2}+\frac{\varepsilon^{2}}{\kappa_{\max }}\|\mathcal{C} \boldsymbol{\tau}\|^{2}+\left(\kappa_{\max }+k_{\max }\right)\|\mathbf{v}\|^{2}+\left(\kappa_{\max }+\varepsilon^{2}\right)\|\mathcal{B} \mathbf{v}\|^{2}+\frac{1}{\kappa_{\max }}\|q\|^{2}\right)^{\frac{1}{2}} \cdot
\end{gathered}
$$

Lemma 2.4. Under the hypothesis of Theorem 2.2, there exists a constant $\alpha(\Omega)$, depending only on the Poincaré constants $\gamma$ and $\beta$ in (1.4), such that for any $(\boldsymbol{\sigma}, \mathbf{u}, p) \in$ $\mathbf{Q}_{w} \times \mathbf{R}_{w} \times W_{w}$, there exists a triplet $(\boldsymbol{\tau}, \mathbf{v}, q) \in \mathbf{Q}_{w} \times \mathbf{R}_{w} \times W_{w}$ such that

$\mathfrak{B}(\boldsymbol{\sigma}, \mathbf{u}, p ; \boldsymbol{\tau}, \mathbf{v}, q) \geq \alpha(\Omega)\left(\|\boldsymbol{\sigma}\|_{\mathbf{Q}_{w}}^{2}+\|\mathbf{u}\|_{\mathbf{R}_{w}}^{2}+\|p\|_{W_{w}}^{2}\right)^{\frac{1}{2}}\left(\|\boldsymbol{\tau}\|_{\mathbf{Q}_{w}}^{2}+\|\mathbf{v}\|_{\mathbf{R}_{w}}^{2}+\|q\|_{W_{w}}^{2}\right)^{\frac{1}{2}}$.

Proof. By the orthogonal decomposition of $H$ (div), we can write

$$
\mathbf{u}=\mathcal{C} \varphi^{\perp}+\mathbf{u}^{\perp}, \text { where } \varphi^{\perp} \in \mathbf{X}^{\perp} \text { and } \mathbf{u}^{\perp} \in \mathbf{Y}^{\perp} .
$$

Thanks to the orthogonal decompositions and the Poincaré inequalities in (1.4), we have

$$
\left\|\varphi^{\perp}\right\|_{\mathbf{Q}} \leq \gamma\left\|\mathcal{C} \varphi^{\perp}\right\|_{\mathbf{R}} \leq \gamma\|\mathbf{u}\|_{\mathbf{R}}, \quad\left\|\mathbf{u}^{\perp}\right\|_{\mathbf{R}} \leq \beta\|\mathcal{B} \mathbf{u}\| .
$$

In a similar way, using also the exactness of (1.3), we can write $p=\mathcal{B w}^{\perp}$, where $\mathbf{w}^{\perp} \in \mathbf{Y}^{\perp}$ and

$$
\left\|\mathbf{w}^{\perp}\right\|_{\mathbf{R}} \leq \beta\left\|\mathcal{B} \mathbf{w}^{\perp}\right\|=\beta\|p\|_{W} .
$$


For some positive real numbers $a_{i}, i=1,2,3,4$ (to be specified later on), we choose

$$
\boldsymbol{\tau}=\boldsymbol{\sigma}-a_{1} \boldsymbol{\varphi}^{\perp} \in \mathbf{Q}, \quad \mathbf{v}=-\mathbf{u}-a_{2} \mathcal{C} \boldsymbol{\sigma}+a_{3} \mathbf{w}^{\perp} \in \mathbf{R}, \quad q=p+a_{4} \mathcal{B} \mathbf{u} \in W .
$$

By direct substitution, we obtain

$$
\begin{array}{r}
\boldsymbol{B}(\boldsymbol{\sigma}, \mathbf{u}, p ; \boldsymbol{\tau}, \mathbf{v}, q)=\|\boldsymbol{\sigma}\|^{2}-a_{1}\left(\boldsymbol{\sigma}, \boldsymbol{\varphi}^{\perp}\right)-\varepsilon(\mathbf{u}, \mathcal{C} \boldsymbol{\sigma})+\varepsilon a_{1}\left(\mathbf{u}, \mathcal{C} \boldsymbol{\varphi}^{\perp}\right) \\
+\varepsilon(\mathcal{C} \boldsymbol{\sigma}, \mathbf{u})+\varepsilon a_{2}\|\mathcal{C} \boldsymbol{\sigma}\|^{2}-\varepsilon a_{3}\left(\mathcal{C} \boldsymbol{\sigma}, \mathbf{w}^{\perp}\right)+\|\sqrt{k(\mathbf{x})} \mathbf{u}\|^{2}+a_{2}(k(\mathbf{x}) \mathbf{u}, \mathcal{C} \boldsymbol{\sigma})-a_{3}\left(k(\mathbf{x}) \mathbf{u}, \mathbf{w}^{\perp}\right) \\
+\varepsilon^{2}\|\mathcal{B} \mathbf{u}\|^{2}+\varepsilon^{2} a_{2}(\mathcal{B} \mathbf{u}, \mathcal{B C} \sigma)-\varepsilon^{2} a_{3}\left(\mathcal{B} \mathbf{u}, \mathcal{B} \mathbf{w}^{\perp}\right)-(p, \mathcal{B} \mathbf{u})-a_{2}(p, \mathcal{B C} \boldsymbol{\sigma})+a_{3}\left(p, \mathcal{B} \mathbf{w}^{\perp}\right) \\
+(\mathcal{B} \mathbf{u}, p)+a_{4}\|\mathcal{B} \mathbf{u}\|^{2}
\end{array}
$$

Exploiting orthogonality and obvious simplifications, noticing that $\left(\mathbf{u}, \mathcal{C} \varphi^{\perp}\right)=\left\|\mathcal{C} \varphi^{\perp}\right\|^{2}$, the above expression reduces to

$$
\begin{gathered}
\mathfrak{B}(\boldsymbol{\sigma}, \mathbf{u}, p ; \boldsymbol{\tau}, \mathbf{v}, q)=\|\boldsymbol{\sigma}\|^{2}+\varepsilon a_{1}\left\|\mathcal{C} \varphi^{\perp}\right\|^{2}-a_{1}\left(\boldsymbol{\sigma}, \boldsymbol{\varphi}^{\perp}\right) \\
+\varepsilon a_{2}\|\mathcal{C} \boldsymbol{\sigma}\|^{2}+\|\sqrt{k(\mathbf{x})} \mathbf{u}\|^{2}+a_{2}(k(\mathbf{x}) \mathbf{u}, \mathcal{C} \boldsymbol{\sigma})-a_{3}\left(k(\mathbf{x}) \mathbf{u}, \mathbf{w}^{\perp}\right) \\
+\varepsilon^{2}\|\mathcal{B} \mathbf{u}\|^{2}-\varepsilon^{2} a_{3}(\mathcal{B} \mathbf{u}, p) \\
+a_{3}\|p\|^{2}+a_{4}\|\mathcal{B} \mathbf{u}\|^{2}
\end{gathered}
$$

Applying Cauchy-Schwarz and Young inequalities, and the Poincaré inequalities (2.3), (2.4), the remaining inner products are estimated as follows:

$$
\begin{gathered}
\left|a_{1}\left(\boldsymbol{\sigma}, \boldsymbol{\varphi}^{\perp}\right)\right| \leq \frac{1}{2}\|\boldsymbol{\sigma}\|^{2}+\frac{a_{1}^{2}}{2}\left\|\boldsymbol{\varphi}^{\perp}\right\|^{2} \leq \frac{1}{2}\|\boldsymbol{\sigma}\|^{2}+\frac{a_{1}^{2}}{2} \gamma^{2}\left\|\mathcal{C} \boldsymbol{\varphi}^{\perp}\right\|^{2}, \\
\left|a_{2}(k(\mathbf{x}) \mathbf{u}, \mathcal{C} \boldsymbol{\sigma})\right| \leq \frac{1}{4}\|\sqrt{k(\mathbf{x})} \mathbf{u}\|^{2}+a_{2}^{2}\|\sqrt{k(\mathbf{x})} \mathcal{C} \boldsymbol{\sigma}\|^{2} \leq \frac{1}{4}\|\sqrt{k(\mathbf{x})} \mathbf{u}\|^{2}+a_{2}^{2} k_{\max }\|\mathcal{C} \boldsymbol{\sigma}\|^{2} \\
\left|a_{3}\left(k(\mathbf{x}) \mathbf{u}, \mathbf{w}^{\perp}\right)\right| \leq \frac{1}{4}\|\sqrt{k(\mathbf{x})} \mathbf{u}\|^{2}+a_{3}^{2}\left\|\sqrt{k(\mathbf{x})} \mathbf{w}^{\perp}\right\|^{2} \leq \frac{1}{4}\|\sqrt{k(\mathbf{x})} \mathbf{u}\|^{2}+a_{3}^{2} k_{\max } \beta^{2}\|p\|^{2}, \\
\left|\varepsilon^{2} a_{3}(\mathcal{B} \mathbf{u}, p)\right| \leq \varepsilon^{2}\|\mathcal{B} \mathbf{u}\|^{2}+\frac{\varepsilon^{2}}{4} a_{3}^{2}\|p\|^{2} .
\end{gathered}
$$

Substituting the above inequalities in the expression for $\mathfrak{B}$ we have

$$
\begin{aligned}
\mathfrak{B}(\boldsymbol{\sigma}, \mathbf{u}, p ; \boldsymbol{\tau}, \mathbf{v}, q) & \geq \frac{1}{2}\|\boldsymbol{\sigma}\|^{2}+\left(\varepsilon a_{2}-a_{2}^{2} k_{\max }\right)\|\mathcal{C} \boldsymbol{\sigma}\|^{2} \\
+\frac{k_{\min }}{2}\|\mathbf{u}\|^{2}+\left(\varepsilon a_{1}-\frac{a_{1}^{2}}{2} \gamma^{2}\right)\left\|\mathcal{C} \boldsymbol{\varphi}^{\perp}\right\|^{2}+a_{4}\|\mathcal{B} \mathbf{u}\|^{2} & \\
& +\left(a_{3}-a_{3}^{2}\left(k_{\max } \beta^{2}+\frac{\varepsilon^{2}}{4}\right)\right)\|p\|^{2} .
\end{aligned}
$$

Then we choose $a_{1}=\frac{\varepsilon}{\gamma^{2}}=\mathcal{O}(\varepsilon), a_{2}=\frac{\varepsilon}{2 k_{\max }+\varepsilon^{2}}=\mathcal{O}\left(\frac{\varepsilon}{\kappa_{\max }}\right), a_{3}=\frac{2}{\left(4 k_{\max } \beta^{2}+\varepsilon^{2}\right)}=$ $\mathcal{O}\left(\frac{1}{\kappa_{\max }}\right)$ and $a_{4}=\kappa_{\min }$, so that

$$
\begin{aligned}
\mathfrak{B}(\boldsymbol{\sigma}, \mathbf{u}, p ; \boldsymbol{\tau}, \mathbf{v}, q) \geq & \frac{1}{2}\|\boldsymbol{\sigma}\|^{2}+\frac{\varepsilon^{2}}{4 \kappa_{\max }}\|\mathcal{C} \boldsymbol{\sigma}\|^{2} \\
& +\frac{k_{\min }}{2}\|\mathbf{u}\|^{2}+\frac{\varepsilon^{2}}{2 \gamma^{2}}\left\|\mathcal{C} \boldsymbol{\varphi}^{\perp}\right\|^{2}+\kappa_{\min }\|\mathcal{B} \mathbf{u}\|^{2}+\frac{1}{4 k_{\max } \beta^{2}+\varepsilon^{2}}\|p\|^{2} .
\end{aligned}
$$


Recalling that $\mathbf{u}=\mathbf{u}^{\perp}+\mathcal{C} \varphi^{\perp}$ and by using the second inequality in (2.3), we have

$$
\begin{aligned}
& \frac{k_{\min }}{2}\|\mathbf{u}\|^{2}+\frac{\varepsilon^{2}}{2 \gamma^{2}}\left\|\mathcal{C} \varphi^{\perp}\right\|^{2}+\kappa_{\min }\|\mathcal{B} \mathbf{u}\|^{2} \\
& \geq \frac{k_{\min }}{2}\left(\left\|\mathbf{u}^{\perp}\right\|^{2}+\left\|\mathcal{C} \varphi^{\perp}\right\|^{2}\right)+\frac{\varepsilon^{2}}{2 \gamma^{2}}\left\|\mathcal{C} \varphi^{\perp}\right\|^{2}+\frac{\kappa_{\min }}{2 \beta^{2}}\left\|\mathbf{u}^{\perp}\right\|^{2}+\frac{\kappa_{\min }}{2}\|\mathcal{B} \mathbf{u}\|^{2} \\
& \geq\left(\frac{k_{\min }}{2}+\frac{\kappa_{\min }}{2 \beta^{2}}\right)\left\|\mathbf{u}^{\perp}\right\|^{2}+\left(\frac{k_{\min }}{2}+\frac{\varepsilon^{2}}{2 \gamma^{2}}\right)\left\|\mathcal{C} \varphi^{\perp}\right\|^{2}+\frac{\kappa_{\min }}{2}\|\mathcal{B} \mathbf{u}\|^{2} \\
& =\left(\left(\frac{1}{2}+\frac{1}{2 \beta^{2}}\right) k_{\min }+\frac{\varepsilon^{2}}{2 \beta^{2}}\right)\left\|\mathbf{u}^{\perp}\right\|^{2}+\left(\frac{k_{\min }}{2}+\frac{\varepsilon^{2}}{2 \gamma^{2}}\right)\left\|\mathcal{C} \varphi^{\perp}\right\|^{2}+\frac{\kappa_{\min }}{2}\|\mathcal{B} \mathbf{u}\|^{2} \\
& \geq \frac{1}{2} \min \left(1, \frac{1}{\gamma^{2}}, \frac{1}{\beta^{2}}\right) \kappa_{\min }\left(\left\|\mathbf{u}^{\perp}\right\|^{2}+\left\|\mathcal{C} \varphi^{\perp}\right\|^{2}\right)+\frac{\kappa_{\min }}{2}\|\mathcal{B} \mathbf{u}\|^{2} \\
& =\frac{1}{2} \min \left(1, \frac{1}{\gamma^{2}}, \frac{1}{\beta^{2}}\right) \kappa_{\min }\|\mathbf{u}\|^{2}+\frac{\kappa_{\min }}{2}\|\mathcal{B} \mathbf{u}\|^{2} \\
& \geq \frac{1}{2} \min \left(1, \frac{1}{\gamma^{2}}, \frac{1}{\beta^{2}}\right) \kappa_{\min }\|\mathbf{u}\|_{\mathbf{R}}^{2} .
\end{aligned}
$$

Using this estimate in the above estimate for $\mathfrak{B}$, implies

$$
\begin{array}{r}
\mathfrak{B}(\boldsymbol{\sigma}, \mathbf{u}, p ; \boldsymbol{\tau}, \mathbf{v}, q) \geq \underline{c}(\Omega)\left(\|\boldsymbol{\sigma}\|^{2}+\frac{\varepsilon^{2}}{\kappa_{\max }}\|\mathcal{C} \boldsymbol{\sigma}\|^{2}+\kappa_{\min }\|\mathbf{u}\|_{\mathbf{R}}^{2}+\frac{1}{\kappa_{\max }}\|p\|_{W}^{2}\right) \\
=\underline{c}(\Omega)\left(\|\boldsymbol{\sigma}\|_{\mathbf{Q}_{w}}^{2}+\|\mathbf{u}\|_{\mathbf{R}_{w}}^{2}+\|p\|_{W_{w}}^{2}\right),
\end{array}
$$

where $\underline{c}(\Omega)$ is a constant depending only on the Poincaré constants $\gamma$ and $\beta$.

To conclude, we notice that

$$
\left(\|\boldsymbol{\tau}\|_{\mathbf{Q}_{w}}^{2}+\|\mathbf{v}\|_{\mathbf{R}_{w}}^{2}+\|q\|_{W_{w}}^{2}\right) \leq[\bar{c}(\Omega)]^{2}\left(\|\boldsymbol{\sigma}\|_{\mathbf{Q}_{w}}^{2}+\|\mathbf{u}\|_{\mathbf{R}_{w}}^{2}+\|p\|_{W_{w}}^{2}\right),
$$

where $\bar{c}(\Omega)$ is a constant depending only on the Poincaré constants $\gamma$ and $\beta$. In fact, the following inequalities hold:

$$
\begin{gathered}
\frac{1}{2}\|\boldsymbol{\tau}\|_{\mathbf{Q}_{w}}^{2} \leq\|\boldsymbol{\sigma}\|_{\mathbf{Q}_{w}}^{2}+a_{1}^{2}\left\|\boldsymbol{\varphi}^{\perp}\right\|_{\mathbf{Q}_{w}}^{2} \leq\|\boldsymbol{\sigma}\|_{\mathbf{Q}_{w}}^{2}+\frac{\varepsilon^{2}}{\gamma^{4}}\|\mathbf{u}\|_{\mathbf{R}}^{2} \leq\|\boldsymbol{\sigma}\|_{\mathbf{Q}_{w}}^{2}+\frac{1}{\gamma^{4}}\|\mathbf{u}\|_{\mathbf{R}_{w}}^{2}, \\
\frac{1}{3}\|\mathbf{v}\|_{\mathbf{R}_{w}}^{2} \leq\|\mathbf{u}\|_{\mathbf{R}_{w}}^{2}+a_{2}^{2} \kappa_{\min }\|\mathcal{C} \boldsymbol{\sigma}\|^{2}+a_{3}^{2} \kappa_{\min }\left\|\mathbf{w}^{\perp}\right\|_{\mathbf{R}}^{2} \\
\leq\|\mathbf{u}\|_{\mathbf{R}_{w}}^{2}+\frac{\kappa_{\min } \varepsilon^{2}}{\kappa_{\max }^{2}}\|\mathcal{C} \boldsymbol{\sigma}\|^{2}+c(\beta) \frac{\kappa_{\min }}{\kappa_{\max }^{2}}\|p\|_{W}^{2} \\
\leq\|\mathbf{u}\|_{\mathbf{R}_{w}}^{2}+\frac{\kappa_{\min }}{\kappa_{\max }}\|\boldsymbol{\sigma}\|_{\mathbf{Q}_{w}}^{2}+c(\beta) \frac{\kappa_{\min }}{\kappa_{\max }}\|p\|_{W_{w}}^{2}, \\
\frac{1}{2}\|q\|_{W_{w}}^{2} \leq\|p\|_{W_{w}}^{2}+a_{4}^{2} \frac{1}{\kappa_{\max }}\|\mathcal{B} \mathbf{u}\|^{2} \leq\|p\|_{W_{w}}^{2}+\frac{\kappa_{\min }^{2}}{\kappa_{\max }}\|\mathbf{u}\|_{\mathbf{R}}^{2} \leq\|p\|_{W_{w}}^{2}+\frac{\kappa_{\min }}{\kappa_{\max }}\|\mathbf{u}\|_{\mathbf{R}_{w}}^{2} .
\end{gathered}
$$

Above, $c(\beta)=\frac{4 \beta^{2}}{\min ^{2}\left(4 \beta^{2}, 1\right)}$. Therefore the inf-sup condition for $\mathfrak{B}$ follows by taking $\alpha(\Omega)=\underline{c}(\Omega) \bar{c}(\Omega)$.

3. Discretization. In this section, we discuss the discretization of the complete de Rham complex, even though the Brinkman problem explicitly involves only the last three spaces of the sequence. In order to achieve stability of the discretized problem, the discrete spaces $S_{h} \subset H^{1}(\Omega), \mathbf{Q}_{h} \subset \mathbf{Q}, \mathbf{R}_{h} \subset \mathbf{R}, W_{h} \subset W$ should preserve the de Rham complex structure of the continuous case (see $[7,8]$ for more details):

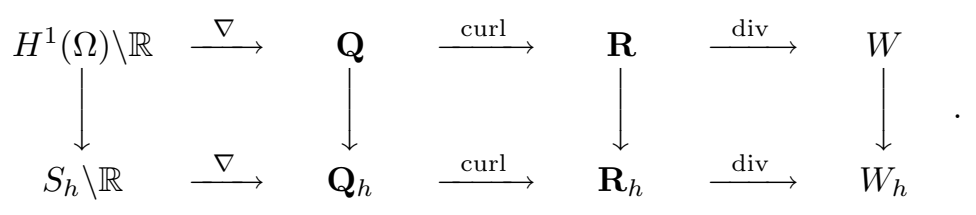


A standard choice for numerical discretization of the Hodge Laplacian is the following. For a given integer $r \geq 0$, we let $S_{h}$ be the continuous piecewise polynomial of degree at most $r+1, \mathbf{Q}_{h}$ the $(r+1)$-th order Nédélec finite elements ([28]), $\mathbf{R}_{h}$ the $r$-th order Raviart-Thomas finite elements $([32,28])$, and $W_{h}$ the discontinuous piecewise polynomials finite element of degree at most $r$.

In fact, this choice of finite elements guarantees that the commutativity property

$$
\operatorname{curl} \Pi_{h}^{\mathbf{Q}}=\Pi_{h}^{\mathbf{R}} \text { curl, and } \operatorname{div} \Pi_{h}^{\mathbf{R}}=\Pi_{h}^{W} \operatorname{div}
$$

holds ([5], [6]). Here, $\Pi_{h}^{\mathbf{V}}: \mathbf{V} \mapsto \mathbf{V}_{h}$ denotes the canonical interpolation operator from the continuous space $\mathbf{V}$ into the finite element counterpart $\mathbf{V}_{h}, \mathbf{V}:=\mathbf{Q}, \mathbf{R}, W$.

Moreover, the above commutativity property together with the exactness of the continuous de Rham complex (for simply connected domain $\Omega$ with simply connected boundary) implies the discrete orthogonal decompositions

$$
\mathbf{R}_{h}=\operatorname{curl} \mathbf{Q}_{h} \oplus \nabla_{h} W_{h}, \text { and } \mathbf{Q}_{h}=\nabla S_{h} \oplus \operatorname{curl}_{h} \mathbf{R}_{h},
$$

where $\nabla_{h}: W_{h} \rightarrow \mathbf{R}_{h}$ is the adjoint of the map - div : $\mathbf{R}_{h} \rightarrow W_{h}$, and $\operatorname{curl}_{h}: \mathbf{R}_{h} \rightarrow$ $\mathbf{Q}_{h}$ is the adjoint map of curl : $\mathbf{Q}_{h} \rightarrow \mathbf{R}_{h}$.

An important result in $[7,8]$ guarantees that the discrete Poincaré inequalities

$$
\text { and } \quad \begin{array}{ll}
\left\|\boldsymbol{\tau}_{h}^{\perp}\right\|_{\mathbf{Q}} \leq \gamma_{h}\left\|\operatorname{curl} \boldsymbol{\tau}_{h}^{\perp}\right\|_{\mathbf{L}^{2}}, & \forall \mathbf{v}_{h}^{\perp} \in \operatorname{curl}_{h} \mathbf{R}_{h}, \\
\text { a } & \leq \beta_{h}\left\|\operatorname{div} \mathbf{v}_{h}^{\perp}\right\|_{L^{2}}, \quad \forall \mathbf{v}_{h}^{\perp} \in \nabla_{h} W_{h}
\end{array}
$$

hold with constants $\gamma_{h}$ and $\beta_{h}$ bounded independently of $h$. Since $\nabla_{h} W_{h} \not \subset \mathbf{Y}^{\perp}$ and $\operatorname{curl}_{h} \mathbf{R}_{h} \not \subset \mathbf{X}^{\perp}$, the above results is not trivial, and it requires the construction of a bounded cochain projector operator $\pi_{h}$ from the continuous to the discrete de Rham complex. Such bounded projectors can be derived by composing the canonical interpolation operators $\Pi_{h}^{\mathbf{V}}$ with commutative smoothing operators ([14]). Then $\gamma_{h} \leq$ $\gamma\left\|\pi_{h}\right\|$ and $\beta_{h} \leq \beta\left\|\pi_{h}\right\|$, where the norm of the cochain projector $\left\|\pi_{h}\right\|$ is uniformly bounded with respect to $h$. We refer to $[7,8]$ for the complete proof.

3.1. Analysis of the discrete problem. We now proceed with the analysis of the finite dimensional approximation of the variational problem, Problem 1.1. The Galerkin formulation of the problem reads

Problem 3.1. Find $\left(\boldsymbol{\sigma}_{h}, \mathbf{u}_{h}, p_{h}\right) \in \mathbf{Q}_{h} \times \mathbf{R}_{h} \times W_{h}$ such that

$$
\left\{\begin{array}{llll}
m\left(\boldsymbol{\sigma}_{h}, \boldsymbol{\tau}_{h}\right) & -c^{*}\left(\mathbf{u}_{h}, \boldsymbol{\tau}_{h}\right) & =F\left(\boldsymbol{\tau}_{h}\right), & \forall \boldsymbol{\tau}_{h} \in \mathbf{Q}_{h} \\
-c\left(\boldsymbol{\sigma}_{h}, \mathbf{v}_{h}\right) & -a\left(\mathbf{u}_{h}, \mathbf{v}_{h}\right)-d\left(\mathbf{u}_{h}, \mathbf{v}_{h}\right) & +b^{*}\left(p_{h}, \mathbf{v}_{h}\right)=G\left(\mathbf{v}_{h}\right), & \forall \mathbf{v}_{h} \in \mathbf{R}_{h} \\
& b\left(\mathbf{u}_{h}, q_{h}\right) & =H\left(q_{h}\right), & \forall q_{h} \in W_{h}
\end{array}\right.
$$

Stability of the Galerkin problem, Problem 3.1, is equivalent to the inf-sup condition for $\mathfrak{B}$ restricted to the finite element space ([10]), which is stated below.

Lemma 3.1. Under the hypothesis of Theorem 2.2, there exists a constant $\alpha_{h}$, depending only on the constants $\gamma_{h}$ and $\beta_{h}$ in the discrete Poincaré inequalities (3.1), such that for any $\left(\boldsymbol{\sigma}_{h}, \mathbf{u}_{h}, p_{h}\right) \in \mathbf{Q}_{h} \times \mathbf{R}_{h} \times W_{h}$ there is a triplet $\left(\boldsymbol{\tau}_{h}, \mathbf{v}_{h}, q_{h}\right) \in \mathbf{Q}_{h} \times$ $\mathbf{R}_{h} \times W_{h}$ for which the following estimate holds:

$$
\begin{aligned}
& \mathfrak{B}\left(\boldsymbol{\sigma}_{h}, \mathbf{u}_{h}, p_{h} ; \boldsymbol{\tau}_{h}, \mathbf{v}_{h}, q_{h}\right) \geq \\
& \alpha_{h}\left(\left\|\boldsymbol{\sigma}_{h}\right\|_{\mathbf{Q}_{w}}^{2}+\left\|\mathbf{u}_{h}\right\|_{\mathbf{R}_{w}}^{2}+\left\|p_{h}\right\|_{W_{w}}^{2}\right)^{\frac{1}{2}}\left(\left\|\boldsymbol{\tau}_{h}\right\|_{\mathbf{Q}_{w}}^{2}+\left\|\mathbf{v}_{h}\right\|_{\mathbf{R}_{w}}^{2}+\left\|q_{h}\right\|_{W_{w}}^{2}\right)^{\frac{1}{2}} .
\end{aligned}
$$


Proof. The proof in the discrete case closely follows the proof given before for the continuous case in Lemma 2.4. Given $\mathbf{u}_{h} \in \mathbf{R}_{h}$ there exists $\boldsymbol{\varphi}_{h}^{\perp} \in \operatorname{curl}_{h} \mathbf{R}_{h} \subset \mathbf{Q}_{h}$ and $\mathbf{u}_{h}^{\perp} \in \nabla_{h} W_{h} \subset \mathbf{R}_{h}$ such that

$$
\mathbf{u}_{h}=\operatorname{curl} \varphi_{h}^{\perp}+\mathbf{u}_{h}^{\perp}, \quad\left\|\varphi_{h}^{\perp}\right\|_{\mathbf{Q}} \leq \gamma_{h}\left\|\operatorname{curl} \varphi^{\perp}\right\|_{\mathbf{R}}, \quad\left\|\mathbf{u}_{h}^{\perp}\right\|_{\mathbf{R}} \leq \beta_{h}\left\|\operatorname{div} \mathbf{u}_{h}\right\| .
$$

Similarly, given $p_{h} \in W_{h}$, there exists $\mathbf{w}_{h}^{\perp} \in \nabla_{h} W_{h}$, such that $p_{h}=\operatorname{div} \mathbf{w}_{h}^{\perp}$ and $\left\|\mathbf{w}_{h}^{\perp}\right\|_{\mathbf{R}} \leq \beta_{h}\left\|p_{h}\right\|_{W}$. The result now follows by taking

$\boldsymbol{\tau}_{h}=\boldsymbol{\sigma}_{h}-a_{1} \boldsymbol{\varphi}_{h}^{\perp} \in \mathbf{Q}_{h}, \mathbf{v}_{h}=-\mathbf{u}_{h}-a_{2} \operatorname{curl} \boldsymbol{\sigma}_{h}+a_{3} \mathbf{w}_{h}^{\perp} \in \mathbf{R}_{h}, q_{h}=p_{h}+a_{4} \operatorname{div} \mathbf{u}_{h}^{\perp} \in W_{h}$

instead of (2.5), and by using the discrete Poincaré inequalities (3.1) instead of the continuous ones (1.4).

The convergence of the discrete solution to the continuous one directly follows from the stability and consistency of the discrete problem.

THEOREM 3.2. Let $(\boldsymbol{\sigma}, \mathbf{u}, p) \in \mathbf{Q} \times \mathbf{R} \times W$ be the solution of the continuous Problem 1.1 and let $\left(\boldsymbol{\sigma}_{h}, \mathbf{u}_{h}, p_{h}\right) \in \mathbf{Q}_{h} \times \mathbf{R}_{h} \times W_{h}$ be the solution of the discrete Problem 3.1. Then

$$
\begin{aligned}
& \left\|\boldsymbol{\sigma}-\boldsymbol{\sigma}_{h}\right\|_{\mathbf{Q}_{w}}^{2}+\left\|\mathbf{u}-\mathbf{u}_{h}\right\|_{\mathbf{R}_{w}}^{2}+\left\|p-p_{h}\right\|_{W_{w}}^{2} \leq \\
& \left(1+\frac{M}{\alpha_{h}} \frac{\kappa_{\max }}{\kappa_{\min }}\right)^{2}\left(\inf _{\boldsymbol{\tau}_{h} \in \mathbf{Q}_{h}}\left\|\boldsymbol{\sigma}-\boldsymbol{\tau}_{h}\right\|_{\mathbf{Q}_{w}}^{2}+\inf _{\mathbf{v}_{h} \in \mathbf{R}_{h}}\left\|\mathbf{u}-\mathbf{v}_{h}\right\|_{\mathbf{R}_{w}}^{2}+\inf _{q_{h} \in W_{h}}\left\|p-q_{h}\right\|_{W_{w}}^{2}\right) .
\end{aligned}
$$

Proof. The proof of this theorem is a direct application of the Babuška Theorem in [9], and follows from the discrete inf-sup condition in Lemma 3.1 and the boundedness of the bilinear form $\mathfrak{B}$ in Lemma 2.3.

Assuming certain smoothness of the continuous solutions and some regularity of the finite element mesh, the above theorem implies the standard error estimate

$$
\left\|\boldsymbol{\sigma}-\boldsymbol{\sigma}_{h}\right\|_{\mathbf{Q}_{w}}^{2}+\left\|\mathbf{u}-\mathbf{u}_{h}\right\|_{\mathbf{R}_{w}}^{2}+\left\|p-p_{h}\right\|_{W_{w}}^{2}=\mathcal{O}\left(h^{r}\right),
$$

for the choice of $(r+1)-t h$ order Nédélec elements, $r$-th order Raviart-Thomas, and $r$-th order piecewise discontinuous elements. We refer to $[17,28,16,3]$ for a detailed analysis of the approximation properties of the Nédélec and Raviart-Thomas spaces.

In the following and in the numerical experiments, we restrict ourselves to the case $r=0$, i.e., to the first order Nédélec space $\mathbf{Q}_{h}$, the lowest order Raviart-Thomas space $\mathbf{R}_{h}$, and the piecewise constant elements, $W_{h}$. Such choice, as well known, leads to discretization error of first order.

4. Preconditioning. The discrete Galerkin problem, Problem 3.1, leads to the solution of a large sparse linear system

$$
\mathcal{B X}=\mathbf{B}
$$

where the block matrix $\mathcal{B}$ and block vectors $\mathbf{X}$ and $\mathbf{B}$ read:

$$
\mathcal{B}=\left[\begin{array}{ccc}
M & -C^{T} & 0 \\
-C & -A-D & B^{T} \\
0 & B & 0
\end{array}\right], \quad \mathbf{X}=\left[\begin{array}{c}
\boldsymbol{\Sigma} \\
\mathbf{U} \\
P
\end{array}\right], \quad \mathbf{B}=\left[\begin{array}{c}
\mathbf{F} \\
\mathbf{G} \\
H
\end{array}\right]
$$


Here $\boldsymbol{\Sigma} \in \mathbb{R}^{\operatorname{dim}\left(\mathbf{Q}_{h}\right)}, \mathbf{U} \in \mathbb{R}^{\operatorname{dim}\left(\mathbf{R}_{h}\right)}, P \in \mathbb{R}^{\operatorname{dim}\left(W_{h}\right)}$ denote the vectors collecting the finite element degrees of freedom $\boldsymbol{\sigma}_{h}^{i}, i=1, \ldots, \operatorname{dim}\left(\mathbf{Q}_{h}\right), \mathbf{u}_{h}^{i}, i=1, \ldots, \operatorname{dim}\left(\mathbf{R}_{h}\right)$ and $p_{h}^{i}, i=1, \ldots, \operatorname{dim}\left(W_{h}\right)$, and $M, C, A, D, B$ are the finite element matrices whose entries are given by

$$
\begin{array}{ll}
M_{i, j}=m\left(\boldsymbol{\sigma}_{h}^{j}, \boldsymbol{\tau}_{h}^{i}\right)=\left(\boldsymbol{\sigma}_{h}^{j}, \boldsymbol{\tau}_{h}^{i}\right), & i, j=1, \ldots, \operatorname{dim}\left(\mathbf{Q}_{h}\right) \\
C_{i, j}=c\left(\boldsymbol{\sigma}_{h}^{j}, \mathbf{v}_{h}^{i}\right)=\varepsilon\left(\operatorname{curl} \boldsymbol{\sigma}_{h}^{j}, \mathbf{v}_{h}^{i}\right), & i=1, \ldots, \operatorname{dim}\left(\mathbf{R}_{h}\right), j=1, \ldots, \operatorname{dim}\left(\mathbf{Q}_{h}\right) \\
A_{i, j}=a\left(\mathbf{u}_{h}^{j}, \mathbf{v}_{h}^{i}\right)=\varepsilon^{2}\left(\operatorname{div} \mathbf{u}_{h}^{j}, \operatorname{div} \mathbf{v}_{h}^{i}\right), & i, j=1, \ldots, \operatorname{dim}\left(\mathbf{R}_{h}\right) \\
D_{i, j}=d\left(\mathbf{u}_{h}^{j}, \mathbf{v}_{h}^{i}\right)=\left(k(\mathbf{x}) \mathbf{u}_{h}^{j}, \mathbf{v}_{h}^{i}\right), & i, j=1, \ldots, \operatorname{dim}\left(\mathbf{R}_{h}\right) \\
B_{i, j}=b\left(\mathbf{u}_{h}^{j}, q_{h}^{i}\right)=\left(\operatorname{div} \mathbf{u}_{h}^{j}, q_{h}^{i}\right), & i=1, \ldots, \operatorname{dim}\left(W_{h}\right), j=1, \ldots, \operatorname{dim}\left(\mathbf{R}_{h}\right) .
\end{array}
$$

The discretized linear system (4.1) has the form of a symmetric saddle-point problem. In fact, if we reorder the unknowns as $(\boldsymbol{\Sigma}, P, \mathbf{U})$, then $\mathcal{B}$ admits the form

$$
\left[\begin{array}{ccc}
M & 0 & -C^{T} \\
0 & 0 & B \\
-C & B^{T} & -(A+D)
\end{array}\right]
$$

It is clear then that $\mathcal{B}$ has $\operatorname{dim}\left(\mathbf{Q}_{h}\right)+\operatorname{dim}\left(W_{h}\right)$ positive eigenvalues and $\operatorname{dim}\left(\mathbf{R}_{h}\right)$ negative eigenvalues. An effective iterative method to solve linear system with symmetric indefinite matrices is minres ([29]) employing a symmetric positive definite preconditioner $\mathcal{P}$.

To derive the preconditioner, we follow the approach presented in ([25]) to precondition symmetric saddle point problems in a functional space setting. According to the authors, the mapping properties of the differential operators of the continuous problem suggest that block diagonal preconditioners are natural choice for saddle point problems. More specifically, given a stability estimate for the continuous problem in some functional spaces, the block diagonal matrix, in which the blocks represent the discretization of the inner products in those spaces, leads to a uniformly bounded (in terms of $h$ ) preconditioner for the saddle point discrete system of interest.

As before, we consider the positive numbers $w_{Q}=\frac{\varepsilon^{2}}{\kappa_{\max }}, w_{R}=\kappa_{\min }, w_{W}=\frac{1}{\kappa_{\max }}$, and introduce the symmetric positive definite variational forms

$$
\begin{array}{lcc}
q\left(\boldsymbol{\sigma}_{h}, \boldsymbol{\tau}_{h}\right)= & \left(\boldsymbol{\sigma}_{h}, \boldsymbol{\tau}_{h}\right)+w_{Q}\left(\operatorname{curl} \boldsymbol{\sigma}_{h}, \operatorname{curl} \boldsymbol{\tau}_{h}\right), & \boldsymbol{\sigma}_{h}, \boldsymbol{\tau}_{h} \in \mathbf{Q}_{h} \\
r\left(\mathbf{u}_{h}, \mathbf{v}_{h}\right)= & w_{R}\left(\mathbf{u}_{h}, \mathbf{v}_{h}\right)+w_{R}\left(\operatorname{div} \mathbf{u}_{h}, \operatorname{div} \mathbf{v}_{h}\right), & \mathbf{u}_{h}, \mathbf{v}_{h} \in \mathbf{R}_{h} \\
w\left(p_{h}, q_{h}\right)= & w_{W}\left(p_{h}, q_{h}\right), &
\end{array}
$$

The above forms define weighted inner products in $\mathbf{Q}_{w}, \mathbf{R}_{w}$ and $W_{w}$.

Therefore Lemma 3.1 and Lemma 2.3, based on [25], imply that a mesh independent preconditioner for the saddle point problem (4.1) is given by

$$
\mathcal{P}=\left[\begin{array}{ccc}
Q & 0 & 0 \\
0 & R & 0 \\
0 & 0 & W
\end{array}\right],
$$

where $Q, R, W$ are the matrix representation of the weighted inner products $q\left(\boldsymbol{\sigma}_{h}, \boldsymbol{\tau}_{h}\right)$, $r\left(\mathbf{u}_{h}, \mathbf{v}_{h}\right)$, and $w\left(p_{h}, q_{h}\right)$ in (4.3). Moreover, letting $K\left(\mathcal{P}^{-1} \mathcal{B}\right)$ denote the spectral condition number of the operator $\mathcal{P}^{-1} \mathcal{B}$, the estimate

$$
K\left(\mathcal{P}^{-1} \mathcal{B}\right) \leq \frac{M}{\alpha_{h}} \frac{\kappa_{\max }}{\kappa_{\min }}
$$


holds with $\mathrm{M}$ in Lemma 2.3 and $\alpha_{h}$ in Lemma 3.1, independent of $h$ (see [34] for the details).

In the particular case of constant coefficient $k(\mathbf{x})=k_{0}$, the condition number is independent of both the mesh size and $k_{0}$. However, in the general case of variable coefficient, the number of minres iterations to solve the preconditioned system will still depend on the ratio $\frac{\kappa_{\max }}{\kappa_{\min }}$.

We remark that, to improve efficiency, it is worth to substitute $\mathcal{P}^{-1}$ with a spectrally equivalent operator $\hat{\mathcal{P}}^{-1}$ that is easier to apply. For example, a viable approach is to let $\hat{\mathcal{P}}$ be an auxiliary space AMG preconditioner of $\mathcal{P}$. In the numerical results section, we use these preconditioners to solve the discrete systems needed for computing the discretization error. For a more detailed description of the preconditioners and their parallel performance, we refer to [34].

5. Numerical Results. The numerical results presented in this section are obtained using the finite element library MFEM [http://code.google.com/p/mfem/], developed at Lawrence Livermore National Laboratory (LLNL). MFEM is a general, modular, parallel $\mathrm{C}++$ library for finite element methods research and development. It supports a wide variety of finite element spaces in $2 \mathrm{D}$ and $3 \mathrm{D}$, as well as many bilinear and linear forms defined on them. It includes classes for dealing with various types of triangular, quadrilateral, tetrahedral and hexahedral meshes and their global and local refinement. Parallelization in MFEM is based on MPI, and it leads to high scalability in the finite element assembly procedure. It supports several solvers from the hypre library (http://www.llnl.gov/CASC/hypre/). In particular, in our tests we used the auxiliary space algebraic multigrid solvers for $H$ (curl) and $H$ (div) ([21], $[22])$.

The initial meshes used in our simulation were generated with the unstructured mesh generator netgen [http://www.hpfem.jku.at/netgen/].

The numerical results presented in this section were obtained on hera, a high performance computer at LLNL. Hera has a total of 864 nodes connected by InfiniBand DDR (Mellanox). Each node has 16 AMD Quad-Core Opteron 2.3Ghz CPUs, and $32 \mathrm{~GB}$ of memory. Hera is running CHAOS 4.4, a linux kernel developed at LLNL, specific for high performance computing.

Our code was compiled with the Intel mpiicc and mpiicpc compilers version 11.1.046.

The numerical results in this section aim to verify the accuracy of the mixed formulation. In particular, we analyze three different test cases: the first for a constant inverse permeability coefficient $k(\mathbf{x})=k_{0}$, the second one for a variable but smooth coefficient, and the third one for discontinuous coefficient.

5.1. Discretization error for constant coefficients.. We study the accuracy of the discretization as function of the ratio $\frac{k}{\varepsilon^{2}}$ in the case of constant inverse permeability $k$. Obviously if $\frac{k}{\varepsilon^{2}}=0$ the Brinkman problem reduces to the Stokes problem, while if $\frac{k}{\varepsilon^{2}} \rightarrow \infty$ the Brinkman problem approaches the Darcy limit.

The analytical solution is given by

$$
\begin{gathered}
\boldsymbol{\sigma}_{e x}=\varepsilon \pi\left[\begin{array}{l}
\sin (\pi x) \cos (\pi y)-\cos (\pi z) \sin (\pi x) \\
\sin (\pi y) \cos (\pi z)-\cos (\pi x) \sin (\pi y) \\
\sin (\pi z) \cos (\pi x)-\cos (\pi y) \sin (\pi z)
\end{array}\right], \quad \mathbf{u}_{e x}=\left[\begin{array}{c}
\sin (\pi y) \sin (\pi z) \\
\sin (\pi z) \sin (\pi x) \\
\sin (\pi x) \sin (\pi y)
\end{array}\right], \\
p_{e x}=8.0 \sin (\pi x) \sin (\pi y) \sin (\pi z) .
\end{gathered}
$$




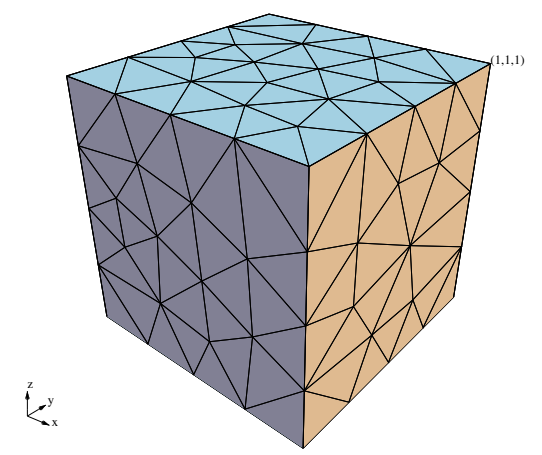

FIG. 5.1. The unstructured mesh used in our accuracy tests.

The right hand side and the natural boundary conditions on $\partial \Omega$ are prescribed accordingly to the analytical solution. To avoid poorly scaled right hand sides, for a given ratio $\frac{k}{\varepsilon^{2}}$ we choose $k$ and $\varepsilon^{2}$ such that $\max \left(k, \varepsilon^{2}\right)=1$.

The domain $\Omega=[0,1]^{3}$ is discretized with an unstructured tetrahedral mesh with 474 elements (Figure 5.1). The original mesh is uniformly refined 5 times, and each element of the mesh is divided in 8 through a bisection algorithm for tetrahedrons. The total number of degrees of freedom ranges from around 2 thousand unknowns on the coarsest mesh up to 65 millions on the finest mesh.

In Figure 5.2 we show the relative discretization errors in the $H(\operatorname{curl} ; \Omega), H(\operatorname{div} ; \Omega)$ and $L^{2}(\Omega)$ norms. The systems were solved by using preconditioned minres with a stopping criterion based on the relative residual norm. As expected from the theory, $\mathbf{u}_{h}$ linearly converges to $\mathbf{u}_{e x}$ in the $H(\operatorname{div} ; \Omega)$ norm and $p_{h}$ linearly converges to $p_{e x}$ in $L^{2}(\Omega)$. Moreover the errors are independent of the ratio $\frac{k}{\varepsilon^{2}}$. The scaled vorticity $\sigma_{h}$, instead, converges linearly to $\sigma_{e x}$ for moderate values of $\frac{k}{\varepsilon^{2}}$, while it shows a degradation in the error behavior for higher values of such ratio.

5.2. Discretization error for non-constant smooth coefficients.. Now we consider the case of non-constant coefficient $k(\mathbf{x})$. For $\Omega=[0,1]^{3}$ and $c \leq 1$ being a positive number, we take

$$
k(\mathbf{x})=\frac{1}{\sin (\pi y) \sin (\pi z)+c} \quad \forall \mathbf{x}=(x, y, z) \in \Omega .
$$

The number $c$ controls how large are the variations in the coefficient $k(\mathbf{x})$, since $k(\mathbf{x})$ ranges between $k_{\min } \sim 1$ and $k_{\max } \sim \frac{1}{c}$. We let the viscosity $\nu=\varepsilon^{2}=1$ and we choose the right hand side and the natural boundary conditions on $\partial \Omega$ be such that the analytical solution is given by

$$
\boldsymbol{\sigma}_{\text {exact }}=\left[\begin{array}{c}
0 \\
\pi \sin (\pi y) \cos (\pi z) \\
-\pi \cos (\pi y) \sin (\pi x)
\end{array}\right], \quad \mathbf{u}_{\text {exact }}=\left[\begin{array}{c}
\sin (\pi y) \sin (\pi z) \\
0 \\
0
\end{array}\right], \quad p_{\text {exact }}=-x .
$$

In this test we use the same initial mesh and refinement strategy as in the previous case of constant coefficients. 

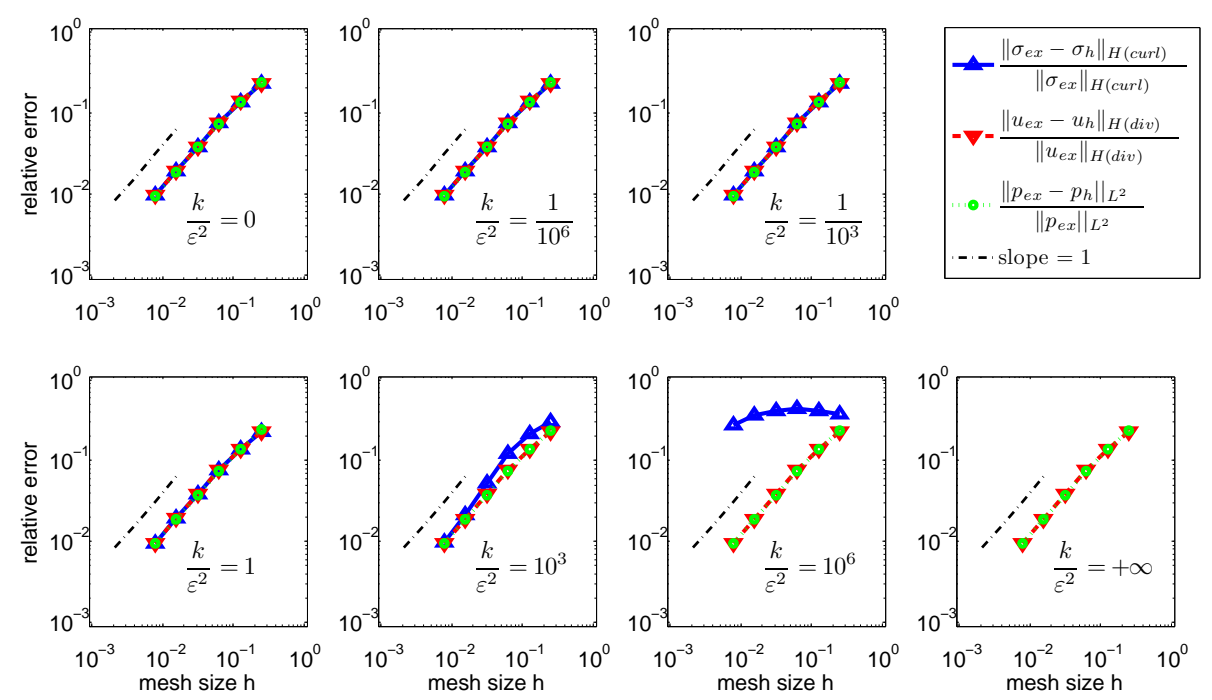

FIG. 5.2. Relative discretization error in the case of constant coefficients for different choices of the ratio $\frac{k}{\varepsilon^{2}}$.
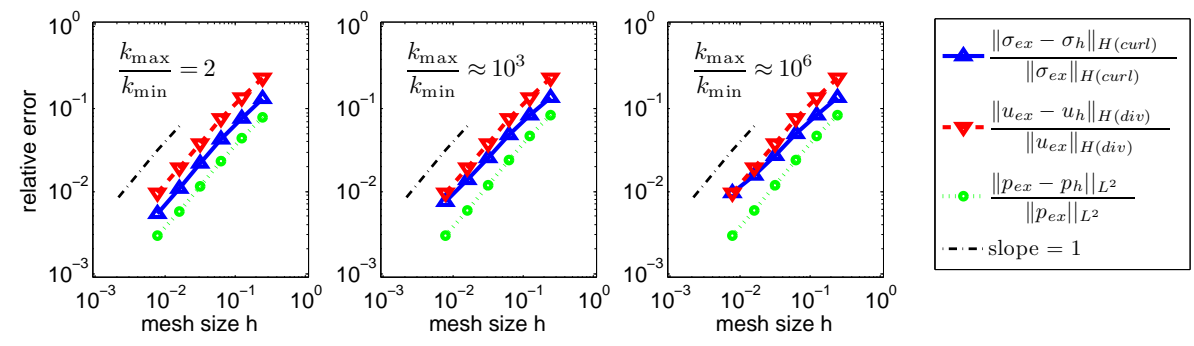

FIG. 5.3. Relative discretization error in the non case of constant coefficients for different choices of the ratio $\frac{k_{\max }}{k_{\min }}$.

In Figure 5.3 we report the relative discretization error with respect to the analytical solution. In all cases, we observe a linear error decay in the $H(\operatorname{curl} ; \Omega)-$ $H(\operatorname{div} ; \Omega)-L^{2}(\Omega)$ energy norms of vorticity, velocity, and pressure.

5.3. Discretization error for coefficients with jumps. For this test we consider the analytical solution of the so-called circular preferential flow pathway proposed in [20]. Such solution describes the steady flow of an incompressible fluid through a circular channel of radius $R$ and length $L$ in an infinite porous medium in response to a constant pressure gradient $\frac{\Delta p}{L}$ in the direction of the channel. Inside the preferential channel the inverse permeability is 0 (Stokes equations), outside is constant and equal to $k$. Using a cylindrical coordinate system, $r$ stands for the distance from the centerline of the preferential channel, $\theta$ is the angle, and $x$ is the coordinate along the centerline; $\hat{\mathbf{r}}, \hat{\boldsymbol{\theta}}, \hat{\mathbf{x}}$ are the unit vector in the radial, tangential, 
and centerline directions. The analytical solution of the flow is given by

$$
\begin{gathered}
\boldsymbol{\sigma}= \begin{cases}\frac{\Delta p}{4 \mu L}(-2 r) \hat{\boldsymbol{\theta}} & \text { if } r \leq R \\
\frac{\Delta p}{4 \mu L}\left(2 R \frac{K_{0}^{\prime}(\sqrt{k} r)}{K_{1}(\sqrt{k} R)}\right) \hat{\boldsymbol{\theta}} & \text { if } r>R,\end{cases} \\
\mathbf{u}= \begin{cases}\frac{\Delta p}{4 \mu L}\left(R^{2}-r^{2}+\frac{4}{k}+\frac{2 R}{\sqrt{k}} \frac{K_{0}(\sqrt{k} R)}{K_{1}(\sqrt{k} R)}\right) \hat{\mathbf{x}} & \text { if } r \leq R \\
\frac{\Delta p}{4 \mu L}\left(\frac{4}{k}+\frac{2 R}{\sqrt{k}} \frac{K_{0}(\sqrt{k} r)}{K_{1}(\sqrt{k} R)}\right) \hat{\mathbf{x}} & \text { if } r>R,\end{cases} \\
p=\frac{\Delta p}{2 L}-\frac{\Delta p}{L} x,
\end{gathered}
$$

where $K_{0}, K_{1}$ are the modified Bessel functions of II type.

In Figure 5.4, we show the velocity and vorticity profiles in the radial direction. The velocity is continuous and differentiable with respect to $r$ for each value of $k$, while the vorticity has a jump in the radial derivative at the interface between the preferential channel and the porous medium $(r=R)$. Moreover for large value of $k$, we observe a boundary layer in the porous medium next to the interface with the preferential channel. In Figure 5.5, we show the three-dimensional solution computed on the finest mesh.

The geometry for this test is a cylinder of radius 2 and length $L=1$. Inside this cylinder we embed a cylinder of radius $R=1$ representing the preferential channel. To improve accuracy, element faces are oriented as the interface between the preferential channel and the porous medium so that no element belongs to both regions. The total number of degrees of freedom ranges from three thousand on the coarser level up to 10 millions on the finest one.

In Figure 5.6, we show the behavior of the discretization error for different values of the inverse permeability $k$ in the porous medium surrounding the preferential channel. We observe that the discretization errors in the $H(\operatorname{div} ; \Omega)$-norm of the velocity and $L^{2}(\Omega)$-norm of the pressure are optimal with respect to the choice of finite elements $(\mathcal{O}(h))$. Similarly to what we already observed in the constant coefficient case, the $H(\operatorname{curl} ; \Omega)$ norm of the discretization error for the vorticity field admits linear decay only for moderate values of the inverse permeability $k$; while for higher values $\left(k=10^{5}\right)$, we do not have convergence to the analytical solution. Indeed, to correctly capture the steep gradient in the vorticity field, local anisotropic mesh refinement should be used due to the developed boundary layer.

Conclusions. In this paper, we analyzed a mixed formulation of the Brinkman problem by introducing the (scaled) vorticity as an additional unknown. The wellposedness analysis of the mixed formulation was based on the Hodge decomposition, and the numerical stability of the method was guaranteed by an analogous results on the discrete level. The particular choice of Nédélec, Raviart-Thomas and piecewise discontinuous elements, in fact, reproduces the same embedding and mapping properties of the continuous spaces in the finite elements spaces. Differently from penalization methods for the Brinkman problem, our approach provides a conforming discretization by standard finite elements. Discretization errors in the $H(\operatorname{div} ; \Omega)$ norm of the velocity and in the $L^{2}(\Omega)$ norm of the pressure show uniform linear decay rate with respect to the inverse permeability coefficient $k(\mathbf{x})$. Only the (scaled) vorticity 

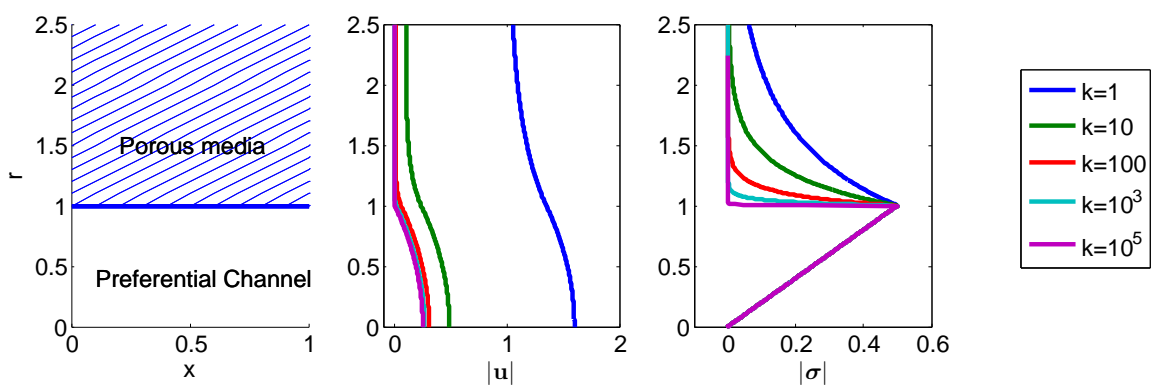

FIG. 5.4. Velocity and vorticity profiles in the radial direction for different values of $k$.
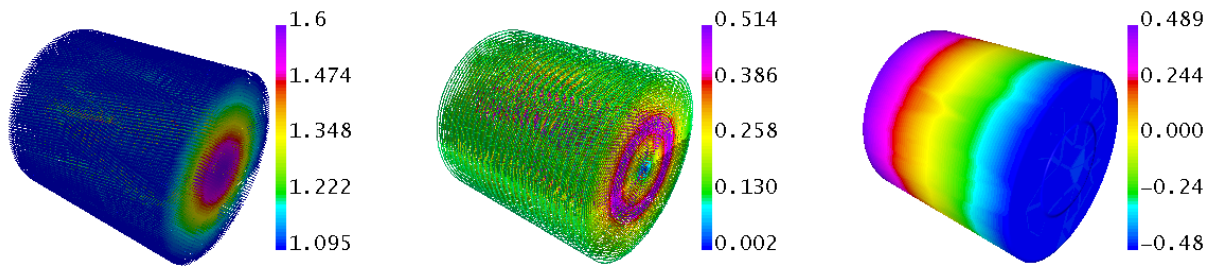

FIG. 5.5. Numerical solution of the preferential channel on the finest grid $(k=1)$ : velocity on the left, vorticity in the center, pressure on the right.

is approximated with less accuracy as we approach the Darcy limit.

Despite the increased number of unknowns, the algebraic linear system obtained after the finite element discretization can be efficiently solved with Krylov iterative methods using block diagonal AMG preconditioning (demonstrated in [34]). A disadvantage of the mixed formulation proposed in this paper is that the Hodge decomposition holds only for particular sets of boundary conditions. As proved in [4], without the Hilbert complex structure, the numerical method is less accurate. Future developments of interest include upscaling techniques and construction of a coarse hierarchy that respects the de Rham complex with good approximation properties, to handle the non-constant coefficient case with both upscaling and solver (multigrid) purpose. The latter is feasible based on appropriate element-based algebraic multigrid (AMGe) technique. For some progress in that direction, we refer to [30], [23]. Finally, in the presence of stable hierarchy of coarse spaces, for stochastic coefficients, multilevel Monte-Carlo process can be employed in order to perform numerical simulations for underground flow of practical interest.

\section{REFERENCES}

[1] G. Allaire, Homogenization of the Navier-Stokes equations in open sets perforated with tiny holes I. Abstract framework, a volume distribution of holes, Archive for Rational Mechanics and Analysis, 113 (1991), pp. 209-259. 10.1007/BF00375065.

[2] — Homogenization of the Navier-Stokes equations in open sets perforated with tiny holes II: Non-critical sizes of the holes for a volume distribution and a surface distribution of holes, Archive for Rational Mechanics and Analysis, 113 (1991), pp. 261-298. 10.1007 /BF00375066.

[3] A. Alonso And A. Valli, An optimal domain decomposition preconditioner for low-frequency time-harmonic Maxwell equations, Mathematics of Computation, 68 (1999), pp. 607-631. 

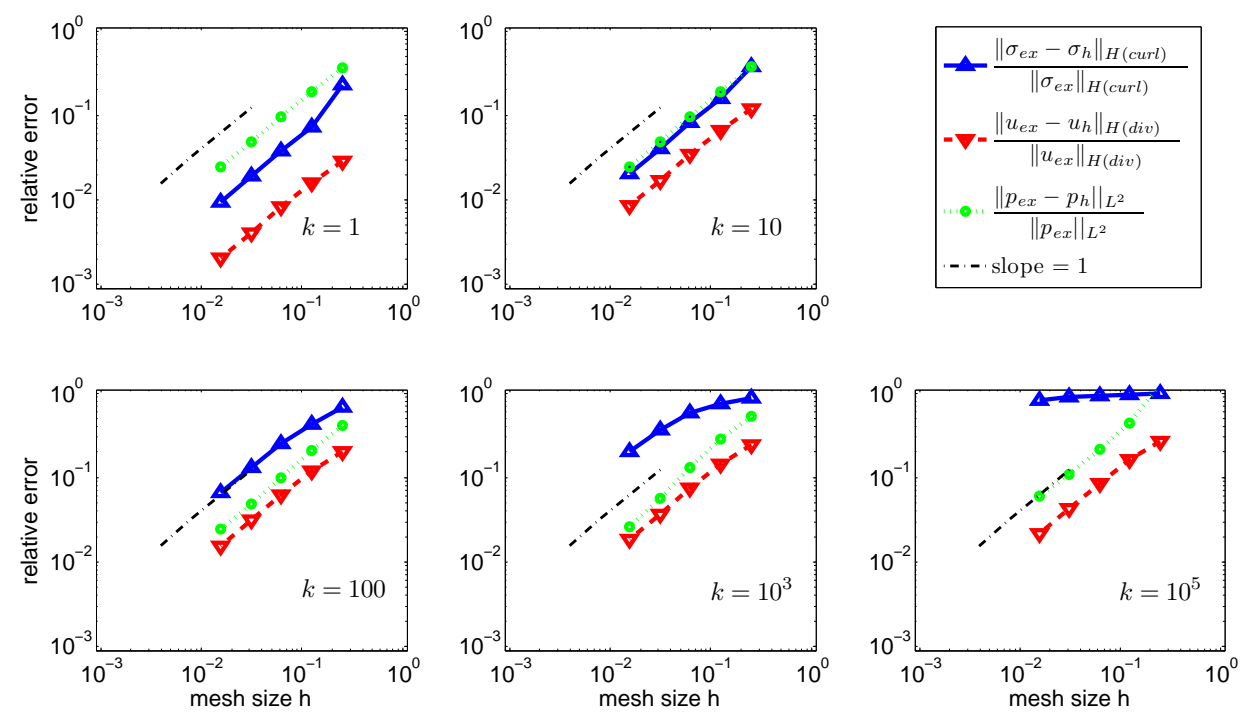

FIG. 5.6. Relative discretization error for the preferential channel test case for different values of the inverse permeability $k$ in the surrounding porous medium.

[4] D. N. Arnold, R. S. Falk, and J. Gopalakrishnan, Mixed finite element approximation of the vector Laplacian with Dirichlet boundary conditions. arXiv 1109.3668, submitted to Mathematical Models \& Methods in Applied Sciences, 2011.

[5] D. N. Arnold, R. S. Falk, And R. Winther, Preconditioning in $H$ (div) and applications, Mathematics of Computations, 66 (1997), pp. 957 - 984.

[6] - Multigrid in H(div) and H(curl), Numerische Mathematik, 85 (2000), pp. 197 - 217.

[7] — Finite element exterior calculus, homological techniques, and applications, Acta Numerica, 15 (2006), pp. 1-155.

[8] - Finite element exterior calculus: from Hodge theory to numerical stability, Bull. Amer. Math. Soc. (N.S.), 47 (2010), pp. 281-354. DOI: 10.1090/S0273-0979-10-01278-4.

[9] I. BABUŠKA, Error-bounds for finite element method, Numer. Math., 16 (1970/1971), pp. 322333.

[10] I. BABUŠKA AND A. K. AzIZ, Survey lectures on the mathematical foundations of finite element method, in The Mathematical Foundations of the FEM with Application to PDE, Academic Press, 1972.

[11] J. Bergh AND J. LÖFStröm, Interpolation spaces: an introduction, Grundlehren der mathematischen Wissenschaften, Springer-Verlag, 1976.

[12] E. Burman And P. Hansbo, Stabilized Crouzeix-Raviart element for the Darcy-Stokes problem, Numerical Methods for Partial Differential Equations, 21 (2005), pp. 986-997.

[13] - A unified stabilized method for Stokes' and Darcy's equations, Journal of Computational and Applied Mathematics, 198 (2007), pp. 35 - 51.

[14] S. H. Christiansen And R. Winther, Smoothed projections in finite element exterior calculus, Mathematics of Computation, 77 (2008), pp. 813-829.

[15] M. R. Correa And A. F. D. Loula, A unified mixed formulation naturally coupling Stokes and Darcy flows, Computer Methods in Applied Mechanics and Engineering, 198 (2009), pp. $2710-2722$.

[16] J. J. Douglas and J. E. Roberts, Global estimates for mixed methods for second order elliptic equations, Math. Comp, 44 (1985), pp. 39-52.

[17] R. S. FAlK AND J. E. OsBonn, Error estimates for mixed methods, in MR 592753 (82j:65076), vol. 14 of RAIRO Anal. Numér., 1980, pp. 249-277.

[18] J. Guzmán and M. Neilan, A family of nonconforming elements for the Brinkman problem, IMA Journal of Numerical Analysis, (2012).

[19] hypre : High performance preconditioners. http://www.llnl.gov/CASC/hypre/. 
[20] A. A. Jennings and R. Pisipati, The impact of Brinkman's extension of Darcy's law in the neighborhood of a circular preferential flow pathway, Environmental Modelling and Software with Environment Data News, 14 (1999), pp. 427-435.

[21] T. Kolev and P. S. Vassilevski, Parallel Auxiliary Space AMG for H(curl) Problems, J. of Computational Mathematics, 27 (2009).

[22] — Parallel Auxiliary Space AMG Solver for H(div) Problems. Technical Report LLNLJRNL-520391, December 15, 2011.

[23] I. V. LAshuk and P. S. VAssilevski, Element Agglomeration Coarse Raviart-Thomas Spaces With Improved Approximation Properties, Numerical Linear Algebra with Applications, 19 (2012), pp. 414-426.

[24] K. A. Mardal, X. C. Tai, and R. Winther, A Robust Finite Element Method for DarcyStokes Flow, SIAM J. Numer. Anal., 40 (2002), pp. 1605-1631.

[25] K. A. Mardal and R. Winther, Preconditioning discretizations of systems of partial differential equations, Numerical Linear Algebra with Applications, 18 (2011), pp. 1-40.

[26] A. Milani And R. Picard, Decomposition theorems and their application to non-linear electroand magneto-static boundary value problems, in Partial Differential Equations and Calculus of Variations, Stefan Hildebrandt and Rolf Leis, eds., vol. 1357 of Lecture Notes in Mathematics, Springer Berlin / Heidelberg, 1988, pp. 317-340. 10.1007/BFb0082873.

[27] P. Monk, Finite Element Methods for Maxwell's Equations, Numerical Mathematics and Scientific Computation, Oxford University Press, Oxford, UK, 2003.

[28] J. C. NÉDÉLEC, Mixed finite elements in $R^{3}$, Numerische Mathematik, 35 (1980), pp. 315-341. 10.1007/BF01396415.

[29] C. C. Paige and M. A. Saunders, Solution of sparse indefinite systems of linear equations, SIAM J. Numerical Analysis, (1975), pp. 617-629.

[30] J. E. Pasciak and P. S. Vassilevski, Exact de Rham Sequences of Spaces Defined on Macroelements in Two and Three Spatial Dimensions, SIAM J. on Scientific Computing, 30 (2008), pp. 2427-2446.

[31] R. PICARD, Some decomposition theorems and their application to non-linear potential theory and Hodge theory, Mathematical Methods in the Applied Sciences, 12 (1990), pp. 35-52.

[32] P. A. Raviart and J. M. Thomas, A mixed finite element method for 2nd order elliptic problems, Mathematical Aspects of the Finite Element Method, Lecture Notes in Mathematics, 606 (1977), pp. 292-315.

[33] A. Toselli and O. B. Widlund, Domain Decomposition Methods-Algorithms and Theory, Springer Series in Computational Mathematics, Springer, 2005.

[34] P. S. Vassilevski and U. Villa, A Block-Diagonal Algebraic Multigrid Preconditioner for the Brinkman Problem. Technical Report LLNL-CONF-522863, 2012.

[35] X. P. Xie, J. C. Xu, AND G. R Xue, Uniformly-stable finite element methods for Darcy-StokesBrinkman models, J. Comput. Math., (2008). 